\title{
A label-free assay for sensitive detection of trypsin by using gold nanoclusters as fluorescent probes
}

\author{
Xu Yan', Hongxia Li', Geyu Lu' \\ ${ }^{1}$ State Key Laboratory on Integrated Optoelectronics, College of Electron Science and Engineering, Jilin \\ University, 2699 Qianjin Street, Changchun, 130012, China \\ yanx@jlu.edu.cn; lugy@jlu.edu.cn
}

\begin{abstract}
:
Herein, we designed a convenient and label-free fluorescence nanosensor for sensitive detection of trypsin (TRY). The fluorescence of gold nanoclusters (AuNCs) could be quenched by cytochrome $\mathrm{c}$ (Cyt c) via electron transfer (ET) mechanism. However, TRY specifically catalyzes the hydrolysis of Cyt $\mathrm{c}$ to small peptide fragments, causing sufficient recovery of fluorescent signal. The proposed system displayed a sensitive and selective response to TRY in the range from 0.2 to $100 \mu \mathrm{g} \mathrm{mL}^{-1}$, with a detection limit of $0.08 \mu \mathrm{g} \mathrm{mL} \mathrm{m}^{-1}$. The designed platform has been successfully applied to the accurately detection of TRY activity in human serum samples and urine samples with satisfactory results. Significantly, the sensing probe has been used to construct paper-based test strips for rapid and visual detection of TRY with highly promising, which validates the potential point-of-care application.
\end{abstract}

Key words: Gold nanoclusters, Cytochrome c, Trypsin, Electron transfer, Test strips

\section{Introduction}

Trypsin (TRY) as an important serine protease can catalyze the hydrolysis of some proteins into small pieces. The enzyme which can regulate pancreatic exocrine function served as a reliable and specific biomarker for pancreatitis. Abnormal level of TRY can also cause cystic fibrosis, pancreatic carcinoma and meconium ileus. Therefore, the sensitive analysis of TRY is of great importance for efficient diagnosis and therapeutics of diseases as well as applications in the proteomics area. Fluorescence (FL) sensors as one of the most powerful sensing platform have received considerable attention due to its characteristics of simplicity, rapid, sensitivity and cost-effective. Herein, we designed a facile AuNCs-based sensing platform for the highly accurate detection of TRY with high sensitivity. More importantly, GSH-capped AuNCs can be applied to fabricate portable paper-based test strips for rapid and visual detection of TRY, implying that it has promising potential for pointof-care assay.

\section{Results and Discussion}

Design of AuNCs-Cyt c Platform. In order to design a sensitive system for the determination of TRY, the AuNCs-based sensing platform coupled with the specificity of enzyme was developed. As shown in Figure 1A, the FL intensity of AuNCs at $625 \mathrm{~nm}$ could be significantly quenched by Cyt $c$ through ET process (Red line). Under catalysis of TRY, Cyt C was hydrolyzed into negatively charged heme-peptide fragments, resulting in the weak ET effect and the recovery of system FL (Blue line). To investigate the feasibility of the proposed system for TRY, control experiment in absence of Cyt $c$ were studied in Figure 1B. It can be obviously found that the FL of AuNCs could not be influenced by TRY in the range from 0 to $100 \mu \mathrm{g} \mathrm{mL}^{-1}$. All of the above results successfully indicated that the designed AuNCs-Cyt c system can be utilized to detect TRY.

For better explore of the quenching mechanism caused by Cyt c, corresponding experiments were carried out. The zeta potential of AuNCs and Cyt $c$ were investigated in Figure 1C. The 
AuNCs were negatively charged $(\zeta=-24.18 \mathrm{mV}$ ) while the Cyt $c$ were positively charged $(\zeta=+17.99 \mathrm{mV})$, indicating that the strong electrostatic interaction between AuNCs and Cyt c. Furthermore, Cyt c is a popular electron transfer substance which can efficiently quench the FL of fluorophore. Thus, we systematically investigated the FL quenching of AuNCs caused by Cyt c. As shown in Figure 1D, the FL intensity of system were gradually quenched with the increasing concentration of Cyt c (0$500 \mu \mathrm{g} \mathrm{mL}-1)$. There is a good linear relationship between $\mathrm{F}_{\mathrm{Q}} / \mathrm{F}_{\mathrm{Q}}$ and the logarithm of Cyt $c$ concentration $\left(\mathrm{R}^{2}=0.9947\right)$. To obtain low background and high sensitivity, $20 \mu \mathrm{g} \mathrm{mL}^{-1}$ of Cyt c was chosen for further study.
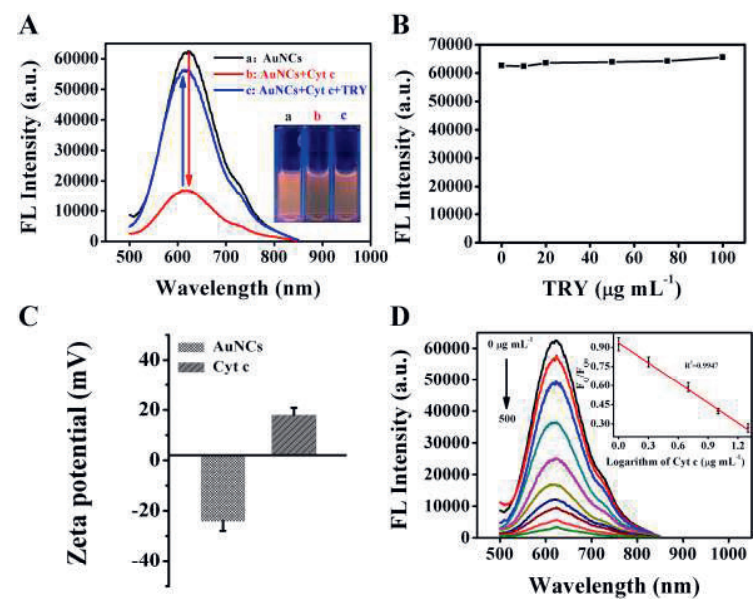

Figure 1 Fluorescence spectra of AuNCs, AuNCsCyt $c$ and AuNCs-Cyt c-TRY. Inset are the corresponding color changes of AuNCs (a), AuNCsCyt c (b) and AuNCs-Cyt c-TRY (c) under UV light. (B) Influence of TRY concentration on the $F L$ intensity of AuNCs. (C) The zeta potentials of AuNCs and Cyt c. (D) FL spectra of AuNCs in the presence of different concentrations of Cyt $c$. Inset are the linear plot of the $F L$ intensity ratio $F_{Q} / F_{Q 0}$ versus the logarithm of Cyt c concentration.

Detection of TRY Activity. As shown in Figure 2A, the FL intensity of AuNCs-Cyt c system was continuously recovered with the increasing of TRY concentration. There is a good linear relationship $\left(R^{2}=0.9916\right)$ between the $F L$ intensity ratio $F_{R} / F_{R 0}$ of AuNCs-Cyt $c$ system and TRY concentration in the range of $0.2-100$ $\mu \mathrm{g} \mathrm{mL}{ }^{-1}$ (Figure 2B). $F_{R}$ and $F_{R O}$ are $F L$ intensities of AuNCs-Cyt $c$ system in the presence and absence of TRY, respectively. The regression equation is: $F_{R} / F_{R 0}=$ $1.0255+0.0240$ [TRY]. The limit of detection (LOD) was calculated to be $0.08 \mu \mathrm{g} \mathrm{mL}^{-1}$ $(3 \sigma / s)$. Furthermore, we proceeded to establish paper-based sensors by immobilization of AuNCs on common absorbent paper. Using the AuNCs-based test paper, we were able to visually detect TRY activity. $10 \mu \mathrm{L}$ of the reaction solution (containing TRY and Cyt c) was dropped on the as-fabricated test strip. With the increase of TRY concentration $(0,2$, $10,20,50$ and $100 \mu \mathrm{g} \mathrm{mL}^{-1}$ ), color of the paper changed from gray to orange with the naked eye under UV light (Figure 2C). These results demonstrated that the AuNCs-based strips sensor offer a highly powerful sensing platform for TRY detection due to their cost-effective, simple operation and ease-transport.

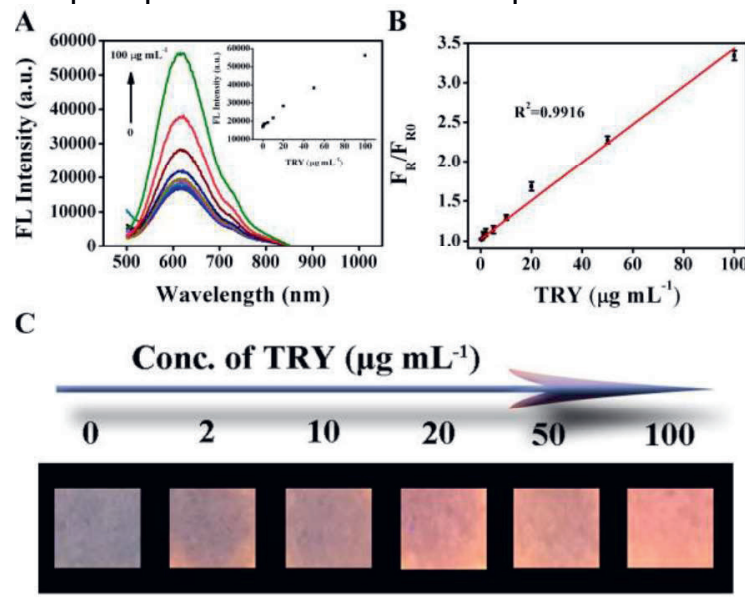

Figure 2 (A) FL spectra of AuNCs-Cyt c sensing probe with different concentrations of TRY. (B) Relationship between FL intensity ratio and the concentration of TRY. Visual detection of TRY by using test strips under a $365 \mathrm{~nm}$ UV illumination. (C) Visual detection of TRY by using test strips under a $365 \mathrm{~nm}$ UV illumination.

\section{Conclusions}

In the present study, a sensitive FL sensing platform has been developed for the sensitive detection of TRY activity by taking advantage of the superior optical properties of GSH capped AuNCs and excellent specificity of TRY. The AuNCs-based FL platform were successfully performed on test strips for visual detection of TRY, which validates the potential point-of-care application. Thus, the proposed probe displays many advantages including environmentalfriendliness, cost-effectiveness, and ease-ofuse, suggesting that the convenient strategy could serve as a promising platform for the detection of TRY.

\section{References}

[1] X. Yan, T. Hu, L. Wang, L. Zhang, X. Su, Nearinfrared fluorescence nanoprobe for enzymesubstrate system sensing and in vitro imaging, Biosens. Bioelectron. 79, 922-929 (2016); doi: 10.1016/j.bios.2016.01.001

[2] X. Yan, H. Li, T. Hu, L. X. Su, A novel fluorimetric sensing platform for highly sensitive detection of organophosphorus pesticides by using egg whiteencapsulated gold nanoclusters, Biosens. Bioelectron. 91, 232-237 (2017); doi: 10.1016/j.bios.2016.11.058 